\title{
Navigating the Google Blind Spot: An Emerging Need for Professional Guidelines to Address Patient-Targeted Googling
}

\author{
Maria J. Baker, Ph.D. 1,2,3, Daniel R. George, Ph.D. ${ }^{3}$, and Gordon L. Kauffman, Jr., M.D. ${ }^{2,3}$ \\ ${ }^{1}$ Penn State Hershey Cancer Institute, Hershey, PA, USA; ${ }^{2}$ Penn State Milton S. Hershey Medical Center, Hershey, PA, USA; ${ }^{3}$ Penn State University \\ College of Medicine, Hershey, PA, USA.
}

\author{
KEY WORDS: doctor-patient relationships; ethics; guidelines; \\ professionalism; patient-targeted googling. \\ J Gen Intern Med 30(1):6-7 \\ DOI: $10.1007 / \mathrm{s} 11606-014-3030-7$ \\ (c) Society of General Internal Medicine 2014
}

$\mathrm{M}$ any physicians would agree that seeking information about their patients via Google seems to be an invasion of privacy, violating trust between patients and their healthcare providers. However, it may be viewed as ethically valid, and even warranted under certain circumstances. Although guidelines developed by the American Medical Association and the Federation of State Medical Boards (FSMB) provide general guidance on the appropriate use of the Internet, they do not specifically address the crucial issue of whether physicians should 'google' their patients, and, if so, under what circumstances. $^{1,2}$ As a result, physicians are left to navigate this "google blind spot" independently, and to decipher on a case-by-case basis where the boundary of professionalism lies with regard to patient-targeted googling.

Two case scenarios illustrate the moral ambiguity present within this "blind spot." The first concerns a female patient who pursued cancer predisposition testing based on her diagnosis of breast cancer at 27 years of age. Testing revealed a mutation in the $B R C A 2$ gene, as well as a $B R C A 2$ variant, the clinical relevance of which had yet to be determined. The patient was counseled about the clinical implications for her risk to develop a new primary breast cancer, ovarian cancer, as well as other BRCA2-associated cancers. Additionally, she was counseled about her reproductive risks and the importance of determining from which side of the family the BRCA2 mutation originated so that at-risk relatives could be notified. Years later, an amended report was received from the genetics laboratory: the variant of uncertain clinical significance had been reclassified as a deleterious mutation, which, together with the patient's BRCA2 mutation previously identified, confirmed a diagnosis of Fanconi anemia, a rare, bone marrow failure disorder. The information in the amended report held important clinical information, not only for the patient's future cancer risks, but also for her relatives on both sides of the family due to its autosomal recessive mode of inheritance. Additionally, her diagnosis of Fanconi anemia conferred a

Published online September 17, 2014
$100 \%$ risk, rather than $50 \%$, for any future offspring to harbor a deleterious $B R C A 2$ mutation. Despite attempts to reach the patient using contact information on file, the clinician was unsuccessful. Given the gravity of the information for the patient, her family members, and future offspring, 'googling' her was viewed as a requirement to fulfill a professional obligation.

The second case involves a 26-year-old female who was requesting bilateral prophylactic mastectomy-without benefit of genetic testing - due to her, almost unbelievable, family history of breast, ovarian, and esophageal cancer. The patient also claimed to have had a melanoma removed, but the pathology report had documented removal of a simple dysplastic nevus. The breast surgeon asked the genetic counselor whether he was on firm ground to perform the requested surgery. Evidently, the patient, in a similar pursuit of prophylactic surgery, had presented to two other facilities with the same request. Due to inconsistencies in the personal and family history information provided, and at the urging of one of the aforementioned outside healthcare providers, the genetic counselor 'googled' the patient to find-via her indexed Facebook profile - that she was presenting her cancer story at lay conferences, giving newspaper interviews, and blogging about her experience as a cancer survivor. Additionally, the patient was raising funds, perhaps fraudulently, to attend a national cancer conference. Armed with this information, the genetic counselor informed the surgeon, who subsequently told the patient he felt uncomfortable performing the surgery in the absence of formal genetic and psychological testing. Following that suggestion, the patient failed to return all subsequent calls.

Both scenarios illustrate situations in which it may be ethically appropriate, and even warranted to 'google' one's patient. In the absence of formal professional guidelines, and based on our experiences with these cases and the recent writing of others on this topic, ${ }^{3}$ we propose the following potential situations that may justify patient-targeted 'googling': 1) duty to re-contact/warn patient of possible harm, 2) evidence of doctor shopping, 3) evasive responses to logical clinical questions, 4) claims in a patient's personal or family history that seem improbable, 5) discrepancies between a patient's verbal history and clinical documentation, 6) levels of urgency/aggressiveness incommensurate with clinical 
assessment, 7) receipt of discrediting information from other reliable health professionals that calls the patient's story into question, 8) dissonant or incongruent statements by the patient, or between a patient and their family members, 9) suspicions regarding physical and/or substance abuse, and 10) concerns regarding suicide risk.

Notably, all of these potential situations, as illustrated by the above case scenarios, are at their core concerned with patient safety. In the first case, there was a moral and ethical duty to notify the patient regarding cancer risks not previously appreciated in her, as well as additional family members, due to reclassification of the uncertain finding. In contrast, the second case unfolded as a result of efforts to prevent the patient from undergoing unnecessary and physically deforming prophylactic surgery, when the request was apparently prompted by a factitious disorder and/or fraudulent intent.

Others who have written on this topic also argue for the role of careful reflection when clinicians undertake patient-targeted 'googling.' Clinton et al. have suggested that, before searching online for a patient, psychiatrists consider various factors such as the intention of the searching, the anticipated effect of gaining information online, and its potential value or risk for the treatment. ${ }^{4}$ Most recently, the American College of Physicians (ACP) and the FSMB encouraged physicians to consider the intent of the search and how it impacts continuing treatment of the patient, while additionally encouraging consideration of how to appropriately document online findings with implications for ongoing care. ${ }^{5}$

Although the AMA Council on Ethical and Judicial Affairs (CEJA) has initiated an important discussion on medical professionalism and the use of social media by publishing Opinion 9.124 and its accompanying policy, as does the policy statement from the ACP and the FSMB, they do not, as yet, comprehensively address the topical issue of patient-targeted 'googling.' Given the growing percentage of U.S. physicians for whom the use of search engines/social media has become daily habit, we propose a pressing need for these documents to be updated, and have within this commentary laid out a foundation for further discussion and debate.
Moreover, with current students in the medical field having come of age on the Internet, and with studies consistently showing that greater than $90 \%$ of medical students actively use social media, overarching guidelines for the use of technology, with specific reference to patient-targeted 'googling,' are required to aid clinical decision-making by future healthcare professionals. We must not only develop useful professional guidelines with relevance to the current environment in which health care providers practice, but we must also address the ethics of patient-targeted 'googling' by incorporating insightful cases involving this practice and social media searches in general into medical professionalism curricula. By doing so, we can help all physicians, nurses, genetic counselors and others navigate this "google blind spot" with greater professional and ethical clarity.

Conflicts of Interest: The authors have no conflicts of interest to disclose.

Corresponding Author: Maria J. Baker, Ph.D.; Penn State University College of Medicine, 500 University Drive, Hershey, PA 17033, USA (e-mail: mbaker@hmc.psu.edu).

\section{REFERENCES}

1. American Medical Association (2010) Professionalism in the use of social media. http://www.ama-assn.org/ama/pub/physician-resources/medicalethics/code-medical-ethics/opinion9124.page. Accessed May 23, 2014.

2. Federation of State Medical Boards. Model policy guidelines for the appropriate use of social media and social networking in medical practice. Euless, TX: Federation of State Medical Boards; April 2012. Accessed at www.fsmb.org/pdf/pub-social-media-guidelines.pdf on May 23, 2014.

3. Warraich HJ. When Doctors 'Google' Their Patients. NY Times, January 6, 2014. Accessed at http://well.blogs.nytimes.com/2014/01/06/when-doctors-google-their-patients-2/?_php=true\&_type=blogs\&_r=0 on May 23, 2014.

4. Clinton BK, Silverman BC, Brendel DH. Patient-targeted googling: the ethics of searching online for patient information. Harv Rev Psychiatry. 2010;18(2):103-12.

5. Farnan JM, Sulmasy LS, Worster BK, Chaudhry HJ, Rhyne JA, Arora VM. Online medical professionalism: patient and public relationships: policy statement from the American College of Physicians and the Federation of State Medical Boards. Ann Intern Med. 2013;158(8):620-7. 